\title{
Oxidative stress and lipid peroxidation may be risk factors for metabolic cardiovascular syndrome in obese prepubertal children
}

\author{
${ }^{1}$ Ghazwan Talal Mehdi, ${ }^{2}$ Prof.Dr.Hedef Dhafir El-Yassin \\ 1 (MSc. Candidate) \\ 2 (PhD. Post Doctorate)/ member of the society for endocrinology, Bristol, UK, Department of Biochemistry, \\ College of Medicine, University of Baghdad.
}

\begin{abstract}
Background: Metabolic cardiovascular syndrome is a combination of factors that augment a person's risk for heart disease, diabetes and stroke. Features of which are obesity, insulin resistance, dyslipidemia [including increased triglycerides and decreased HDL], impaired glucose tolerance, and hypertension. Obesity has the central role in this syndrome. The prevalence of obesity in children has increased dramatically over the last 20 years, leading to higher health risk associated with complications such as type 2 diabetes, high blood pressure and cardiovascular disease.

Aim: The aim of this study is to search for a possible relationship between oxidative stress (as interpreted by lipid peroxidation) and risk factors for metabolic cardiovascular syndrome in obese prepubertal children, in an attempt to overcome the increased health risk associated with obesity in children.
\end{abstract}

Subjects and methods: Eighty prepubertal children aged (5-11) years were enrolled in this study. According to their body mass index (BMI) they were divided into two groups:

1. Obese group of which 50 subjects were selected

2. Lean group consisted 30 aged matched subjects.

Random cholesterol, high density lipoproteins (HDL) and malondialdehyde were measured in sera spectrophotometricaly

Results: Both cholesterol and MDA serum concentrations were significantly higher in obese children than leans $(P<0.000)$. However HDL was lower in obese children and no significant value was found when compared to leans.

Conclusion: Obese children exhibited hypercholesterolemia and increased lipid peroxidation. This might be a potential risk of hypertension and cardiovascular diseases especially when HDL decreases as was found. These findings might shed a light on a possible relationship between oxidative stress metabolic cardiovascular syndrome. A case where interventional measures might be necessary to prevent excessive weight gain during childhood.

\section{Introduction}

Metabolic cardiovascular syndrome is a combination of factors that augment a person's risk for heart disease, diabetes and stroke. Features of which are obesity, insulin resistance, dyslipidemia [including increased triglycerides and decreased HDL], impaired glucose tolerance, and hypertension (1). Obesity has the central role in this syndrome (2). The prevalence of obesity in children has increased dramatically over the last 20 years, leading to higher health risk associated with complications such as type 2 diabetes, high blood pressure and cardiovascular disease.

Studies have suggested that obesity is associated with increased oxidative stress. Analysis of oxidative markers in obesity subjects indicates that oxidative damage is associated with increased BMI and percentage of body fat (3)

The possible mechanisms of obesity related oxidative stress include increased oxygen consumption and subsequent production of free radicals derived from the increase in mitochondrial respiration, diminished antioxidant capacity, fatty acid oxidation, lipid oxidizability, and cell injury causing increased rates of free radical formation. $(4,5)$.

Malondialdehyde (MDA) is one of the most frequently used indicators of lipid peroxidation (6).

Malondialdehyde, MDA, is a highly reactive three carbon dialdehyde produced as a byproduct of a poly unsaturated fatty acid peroxidation. In fact MDA is the principal and most studied product of polyunsaturated fatty acid peroxidation (7).

The aim of this study is to search for a possible relationship between oxidative stress (as interpreted by lipid peroxidation) and risk factors for metabolic cardiovascular syndrome in obese prepubertal children, in an attempt to overcome the increased health risk associated with obesity in children. 


\section{Subjects and methods:}

The study was performed during the period from January 2013 to march 2013. For this purpose 50 healthy obese children and 30 normal weight children were selected from people attended the local teaching hospital and students from some primary schools .the age of children was between 5 to 11 years.

The study was performed with the approval of the medical ethical committee in the ministry of health and ministry of education (Directorate General of education in Diyala). The parents' agreement for blood drawing from their children was also granted.

From each subject, five $\mathrm{ml}$ of venous blood were aspirated from a suitable vein. Sera were obtained after centrifugation at $4000 \mathrm{rpm}$ for 15 minutes. The sera were used for assessing the levels cholesterol, HDL and MDA. Total cholesterol was determined by enzymatic method. Kits were supplied by Bio-Maghreb Company - Tunisia. While kites for HDL determination were supplied by Bio- Labo Company - France.

The concentration of malandialdehyde in serum was determined according to Buege and Aust (1978) by enzymoloical method

Thiobarbituric acid (TBA) method was used to measure the (MDA) which gives a pink color that was read at $(532 \mathrm{~nm})$. Malondialdehyde concentration was calculated by using the molar extinction coefficient of $1.56 \times 10^{5}$.

Anthropometrical Measurements: Body mass index (BMI) is a measure used to determine children's obesity. It is calculated using the child's weight and height. BMI does not measure body fat directly, but it is a reasonable indicator of body fatness for most children and teens.

A child's weight status is determined using an age- and sex-specific percentile for BMI rather than the BMI categories used for adults because children's body composition varies as they age and also varies between boys and girls.

Centers for Disease Control and Prevention (CDC) Growth Charts are used to determine the corresponding BMI-for-age and sex percentile. For children and adolescents (aged 2-19 years):

A- Obesity is defined as a BMI at or above the 95th percentile for children of the same age and sex.

B- Normal is defined as a BMI at or above the 5 th to 85 th percentile for children of the same age and sex .

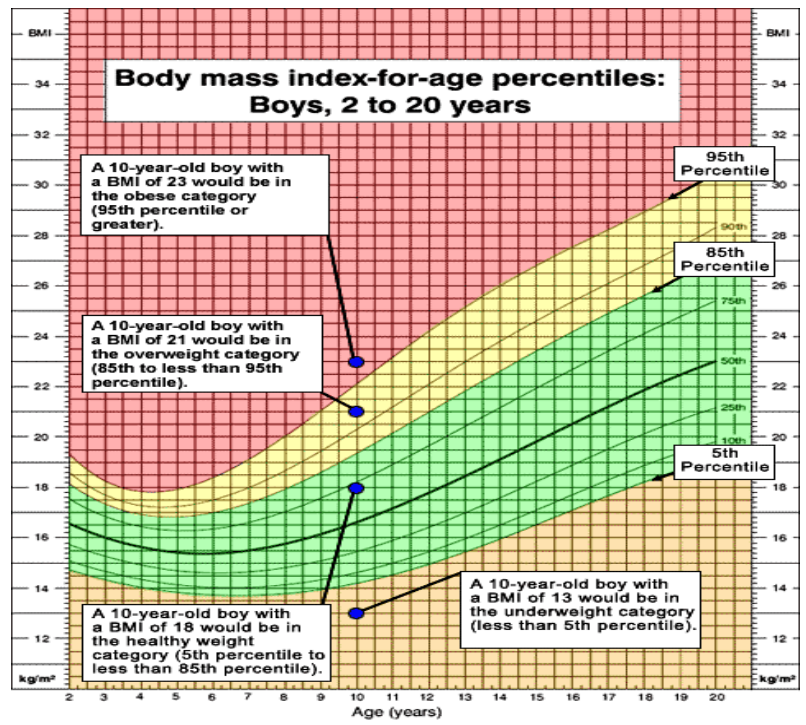

Figure (1): Centers for Disease Control and Prevention (CDC) Growth Charts for male and female children (www.cdc.gov/growthcharts/)

Data were entered and analyzed using SPSS version 16 (SPSS Inc, USA ) for windows ( Microsoft corporation, USA ). Continuous variable were expressed as mean $\pm \mathrm{SD}$, while categorical variable were expressed as a percentage and $95 \%$ CI . T - test, Anova. The level of significance for all statistical test was set at 0.05 . 


\section{Results and discussion:}

Table 1 and figure (2) summarizes the results obtained in both obese and lean weight children. As shown in mean, standard Error of the mean and significance.

Table 1: Mean, standard Error mean, sig (2-tailed) of the Parameters studied in the two groups

\begin{tabular}{|l|c|c|c|}
\hline & $\begin{array}{l}\text { Obese } \\
(\text { Mean } \pm \text { SEM })\end{array}$ & \multicolumn{1}{l|}{$\begin{array}{l}\text { lean } \\
(\text { Mean } \pm \text { SEM })\end{array}$} \\
\hline $\begin{array}{l}\text { Tot. } \\
\text { Cholesterol }\end{array}$ & $166.22 \pm 3.80$ & $144.4 \pm 4.19$ & 0.000 \\
\hline HDL & $51.66 \pm 2.10$ & $53.10 \pm 1.27$ & 0.621 \\
\hline MDA & $0.06 \pm 0.00$ & $0.04 \pm 0.00$ & 0.000 \\
\hline
\end{tabular}

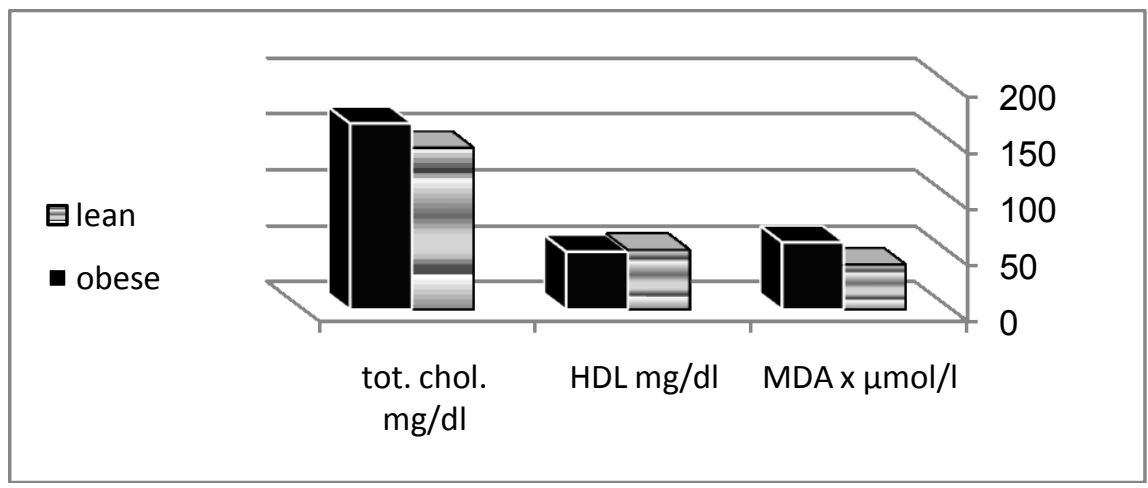

Figure(2) : Mean values of the Parameters studied in the two groups

Both total cholesterol and MDA were elevated significantly in obese subjects when compared to lean ones. On the other hand no significant decrease in HDL was observed between both groups.

The site of increased cholesterol synthesis in obese subjects is of a major interest. It is concluded that plasma cholesterol concentrations does not necessarily reflect cholesterol synthesis rates (8). The possibility that the overall increase in cholesterol turnover might reflect synthesis in adipose tissue, a tissue in which cholesterol turns over more slower than in liver or intestine. Total body cholesterol synthesis is significantly correlated with both excess body weight and adipose cellularity. The known increased flux of free fatty acids in obesity (9) may provide more acetyl CoA precursor for cholesterologenesis.

Both groups shouwed medium levels og HDL being in th obese lower than in lean. It is well known that Low HDL cholesterol, heightened the risk for heart disease and thus obese subject are more susebtible to CVD (10) .

Alterations in the values of MDA is the first evidence of a significant altered oxidant antioxidant status in prepubertal children affected by severe obesity. Previous studies have shown that the mean MDA levels are higher in obese individuals compared to nonobese healthy controls (11). It is also shown that obesity is associated with increases in endogenous lipid peroxides (12). Significant decrease in oxidative stress after dietary restriction and weight loss has been reported (13).

\section{IV. conclusion:}

Obesity causes an oxidative stress by amplifying the lipid peroxidation products. Interaction of oxidative stress with obesity, might lead to complications such as hyperinsulinemia and insulin resistance, thus life threatening results are expected. Serious interventions in the childhood period may prevent future obesity-related oxidative damages.

In the light of these results one should take intervention measures which are necessary to prevent excessive increase in weight during childhood.

\section{References}

[1] Moreno LA, Pineda I, Rodriguez G et al. Leptin and metabolic syndrome in obese and non-obese children. Horm Metab Res 2002; $34: 394-399$.

[2] Chu NF, Wang DJ, Shieh SM et al. Plasma leptin concentrations and obesity in relation to insulin resistance syndrome components among school children in Taiwan-The Taipi Children Heart Study. Int J Obesity 2000; 24 : 1265-1271. 
[3] Pihl E, Zilmer K, Kullisaar T, Kairane C, Mägi A, Zilmer M. Atherogenic inflammatory and oxidative stress markers in relation to overweight values in male former athletes. Int J Obes (Lond). 2006 Jan;30(1):141-6.

[4] J. F. Keaney Jr., M. G. Larson, R. S. Vasan et al., "Obesity and systemic oxidative stress: clinical correlates of oxidative stress in the Framingham study," Arteriosclerosis, Thrombosis, and Vascular Biology, 2003 vol. 23, no. 3, pp. 434-439,

[5] H. K. Vincent and A. G. Taylor, "Biomarkers and potential mechanisms of obesity-induced oxidant stress in humans," International Journal of Obesity, 2006vol. 30, no. 3, pp. 400-418,

[6] Nielsen F. Mikkelsen B.B. Nielsen J.B. et al. plasma malondialdehyde as biomarker for oxidative stress: reference interval and effects of life-style factor. Clin. Chem. (1997) 43:1209.

[7] Rio D.D., Stewart A.J., Pellegrini N. A review of recent studies on malondialdehyde as toxic molecule and biological marker of oxidative stress. Nutr.Metab.Cardiovasc.dis. (2005) 15:316.

[8] Grundy, S. M., E. H. Ahrens, Jr., and G. Salen. Interruption of the enterohepatic circulation of bile acids in man: comparative effects of cholestyramine and ileal exclusion on cholesterol metabolism. J. Lab. Clin. Med.1971. 78: 94.

[9] Nestel, P. J., H. M. Whyte, and DeW. S. Goodman. Distribution and turnover of cholesterol in humans. J. Clin. Invest. 1968. 48: 982 .

[10] Krauss, Ronald; Patricia J Blanche, Robin S Rawlings, Harriett S Fernstrom, and Paul T Williams . "Separate effects of reduced carbohydrate intake and weight loss on atherogenic dyslipidemia". American Journal of Clinical Nutrition 200683 (5): $1025-1031$.

[11] Vehkala L, Ukkola O, Kesäniemi YA, Kähönen M, Nieminen MS, Salomaa V, Jula A, Hörkkö S. Plasma IgA antibody levels to malondialdehyde acetaldehyde-adducts are associated with inflammatory mediators, obesity and type 2 diabetes. Ann Med. 2013 Oct 17.

[12] Ahmad A, Singhal U, Hossain MM, Islam N, Rizvi I. The role of the endogenous antioxidant enzymes and malondialdehyde in essential hypertension. J Clin Diagn Res. 2013 Jun;7(6):987-90.

[13] Dandona P, Mohanty P, Ghanim H, et al. The suppressive effect of dietary restriction and weight loss in the obese on the generation of reactive oxygen species by leukocytes, lipid peroxidation, and protein carbonylation. J Clin Endocrinol Metab 2001, 86: 35 5-362. 